\title{
Scaling properties for a family of discontinuous mappings
}

\author{
J.A. Méndez-Bermúdez ${ }^{\mathrm{a}, *}$, Juliano A. de Oliveira ${ }^{\mathrm{b}}$, R. Aguilar-Sánchez ${ }^{\mathrm{c}}$, \\ Edson D. Leonel ${ }^{\mathrm{d}, \mathrm{e}}$ \\ a Instituto de Física, Benemérita Universidad Autónoma de Puebla, Apartado Postal J-48, Puebla 72570, Mexico \\ b UNESP - Univ Estadual Paulista, Câmpus São João da Boa Vista, São João da Boa Vista, SP 13874-149, Brazil \\ ${ }^{\mathrm{c}}$ Facultad de Ciencias Químicas, Benemérita Universidad Autónoma de Puebla, Puebla 72570, Mexico \\ ${ }^{d}$ Departamento de Física, UNESP - Univ Estadual Paulista, Av. 24A, 1515, Bela Vista, 13506-900 Rio Claro, SP, Brazil \\ e Abdus Salam International Center for Theoretical Physics, Strada Costiera 11, 34151 Trieste, Italy
}

H I G H L I G H T S

- We study scaling properties of a family of nonlinear discontinuous maps.

- This family is the discontinuous-map representation of well-known nonlinear systems.

- The exponent characterizing the family of maps defines universality classes.

\section{A R T I C L E I N F O}

\section{Article history:}

Received 2 February 2015

Received in revised form 5 April 2015

Available online 12 May 2015

\section{Keywords:}

Nonlinear map

Discontinuous function

Scaling laws

\begin{abstract}
A B S T R A C T
Scaling exponents that describe a transition from integrability to non-integrability in a family of two-dimensional, nonlinear, and discontinuous mappings are obtained. The mapping considered is parameterized by the exponent $\gamma$ in the action variable. The scaling exponents describing the behavior of the average square action along the chaotic orbits are obtained for different values of $\gamma$; therefore classes of universality can be defined. For specific values of $\gamma$ our mapping acts as the discontinuous-map representation of well-known nonlinear systems, thus making our study broadly applicable. Also, the formalism used is general and the procedure can be extended to characterize many other dynamical systems.
\end{abstract}

(c) 2015 Elsevier B.V. All rights reserved.

\section{Introduction and model}

Parametric Hamiltonians $H(K)$ appear frequently in the study of physical problems of interest, both in the classical and quantum domains, where the change of $K$ may represent the effect of an external field (electric field, magnetic flux, gate voltage) or a change of an effective interaction (as in many body systems). In general, when the perturbation is small $H(K)$ can be split in two parts: $H_{0}$ representing the unperturbed system, whose dynamics could be integrable or non-integrable, and $H_{1}$ representing the perturbation parameterized by $K$; that is, $H(K)=H_{0}+K H_{1}$.

In particular, for a two-dimensional (2D) classical system given in action-angle variables $H(K)$ takes the form [1]

$$
H\left(K, I_{1}, I_{2}, \theta_{1}, \theta_{2}\right)=H_{0}\left(I_{1}, I_{2}\right)+K H_{1}\left(I_{1}, I_{2}, \theta_{1}, \theta_{2}\right) .
$$

Here, since $H_{0}$ is assumed to be integrable, $K$ controls the transition from integrability to non-integrability. In fact, a very useful approach to study the dynamics of $H(K)$ in Eq. (1) is to consider a Poincaré section defined by the plane $I_{1} \times \theta_{1}$

\footnotetext{
* Corresponding author. Tel.: +52 222 2295610; fax: +52 2222295611.

E-mail address: jmendezb@ifuap.buap.mx (J.A. Méndez-Bermúdez).

URL: http://www.ifuap.buap.mx/ jmendezb (J.A. Méndez-Bermúdez).
} 
taking $\theta_{2}$ as constant $(\bmod 2 \pi)$. Then, the generic $2 \mathrm{D}$ (Poincaré) mapping which qualitatively describes the behavior of $H(K)$ reads [1]

$$
M_{K}:\left\{\begin{array}{l}
I_{n+1}=I_{n}+K f\left(\theta_{n}, I_{n+1}\right), \\
\theta_{n+1}=\left[\theta_{n}+g\left(I_{n+1}\right)+K h\left(\theta_{n}, I_{n+1}\right)\right] \bmod (2 \pi),
\end{array}\right.
$$

where $f, g$, and $h$ are assumed to be nonlinear functions, $n$ is the $n$th iteration of the mapping, and the variables $I$ and $\theta$ correspond indeed to $I_{1}$ and $\theta_{1}$, respectively.

Mapping $M_{K}$ is very general, so depending on the choice of the nonlinear functions $(f, g$, and $h)$ its dynamics may represent a wide range of different physical systems. As examples, we can mention some well known mappings having in common the choice of $f\left(\theta_{n}, I_{n+1}\right)=\sin \left(\theta_{n}\right)$ and $h\left(\theta_{n}, I_{n+1}\right)=0$ : Chirikov's standard map [2,3], $g\left(I_{n+1}\right)=I_{n+1}$, also known as Taylor-Chirikov's map; the bouncer model [4], $g\left(I_{n+1}\right)=\xi I_{n+1}$; the logistic twist map [5], $g\left(I_{n+1}\right)=I_{n+1}+\xi I_{n+1}^{2}$; the Fermi-Ulam accelerator model [6,7], $g\left(I_{n+1}\right)=2 / I_{n+1}$; a generalized Fermi-Ulam accelerator $(F U)$ model [8-11],

$$
g\left(I_{n+1}\right)=\frac{1}{I_{n+1}^{\gamma}} \text { with } 0<\gamma \leq 1
$$

and the hybrid Fermi-Ulam bouncer model [12], $g\left(I_{n+1}\right)=4 \xi^{2}\left[I_{n+1}-\left(I_{n+1}^{2}-\xi^{-2}\right)^{1 / 2}\right]$ if $I_{n+1}>\xi^{-1}$ and $g\left(I_{n+1}\right)=4 \xi^{2} I_{n+1}$ if $I_{n+1} \leq \xi^{-1}$. Even though the functional form of $g(I)$ for the mappings above vary significantly from one map to another, all share a common dynamical feature: the generic transition to chaos ${ }^{1}$ driven by the parameter $K$. In fact, the maps above develop two dynamical regimes separated by the critical parameter $K_{c}$. When $K<K_{c}$, the phase space is composed of stochastic motion bounded by invariant tori, known as KAM (Kolmogorov-Arnold-Moser) scenario [1]. At $K=K_{c}$, the last KAM curve is destroyed and the transition to global stochasticity takes place. Then, for $K>K_{c}$, I becomes unbounded and increases diffusively.

It is relevant to stress that the generic transition to chaos shortly described above mainly relies on the choice of $f\left(\theta_{n}, I_{n+1}\right)$ made for the maps listed above; i.e. $f\left(\theta_{n}, I_{n+1}\right)=\sin \left(\theta_{n}\right)$. As a mater of fact, when choosing $f\left(\theta_{n}, I_{n+1}\right)$ to be the discontinuous function

$$
f\left(\theta_{n}, I_{n+1}\right) \equiv f\left(\theta_{n}\right)=\sin \left(\theta_{n}\right) \operatorname{sgn}\left[\cos \left(\theta_{n}\right)\right],
$$

map $M_{K}$ (with $h\left(\theta_{n}, I_{n+1}\right)=0$ and $\left.g\left(I_{n+1}\right)=I_{n+1}\right)$ also has two different dynamical regimes delimited by the critical value $K_{c}=1$ [15], however for $K<K_{c}, M_{K}$ does not show stability islands. Actually, due to the discontinuities of $f(\theta)$, KAM theorem is not satisfied and map $M_{K}$ does not develop the KAM scenario. Moreover, for any $K \neq 0$ the dynamics of this discontinuous map is diffusive and a single trajectory can explore the entire phase space [15]. Nevertheless, when $K<K_{c}$ the dynamics is far from being stochastic due to the sticking of trajectories along cantori (fragments of KAM invariant tori). Examples of physical systems described by discontinuous maps are 2D billiard models like the stadium billiard [16,17] and polygonal billiards $[18,19]$.

Even though some scaling properties of discontinuous maps have been recently studied $[20,21]$ there is still a huge gap in the understanding of such maps, as compared to maps developing the KAM scenario. Thus, in order to contribute to fill this gap, in this paper we study some dynamical properties of the following discontinuous map ${ }^{2}$ :

$$
M_{\gamma}:\left\{\begin{array}{l}
I_{n+1}=\left|I_{n}-K \sin \left(\theta_{n}\right) \operatorname{sgn}\left[\cos \left(\theta_{n}\right)\right]\right|, \\
\theta_{n+1}=\left[\theta_{n}+\frac{1}{I_{n+1}^{\gamma}}\right] \bmod (2 \pi) \quad \text { with } 0<\gamma \leq 1 .
\end{array}\right.
$$

Notice that map $M_{\gamma}$ is in fact the discontinuous-map version of the $F U$ model [8-11] characterized by the function $g(I)$ given in Eq. (3). We consider here only the case $K<1$. In addition, note that due to parameter $\gamma$ in (5), map $M_{\gamma}$ represents a family of discontinuous maps. ${ }^{3}$

Then, in the following section we will explore and characterize some dynamical properties of map $M_{\gamma}$ when $K<1$. In particular we will focus on the behavior of the average square action $\left\langle I^{2}\right\rangle$ and the average standard deviation of $I$, that we name here $\omega$, as a function of the $n$th iteration of the map as well as the parameters $K$ and $\gamma$.

\footnotetext{
1 The description of the generic transition to chaos, where Chirikov's standard map is used as a paradigm, can be found in well known textbooks [1,13] as well as in recent research papers, see e.g. Ref. [14].

2 The absolute value in the first equation of (5) is necessary to avoid the fractional powers of negative numbers in the equation for the phase $\theta$.

3 In fact, the continuous version of map $M_{\gamma}$ (i.e. the $F U$ model) represents physically relevant systems for specific values of $\gamma$ : For $\gamma=1$, both, the Fermi-Ulam accelerator model [6,7] and the ripple billiard model $[22,23]$ are recovered. For $\gamma=1 / 2$ some dynamical properties of a time-dependent potential well [24] are retrieved.
} 
a

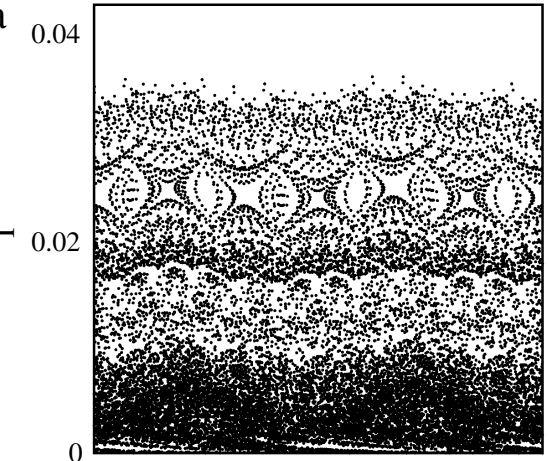

$\mathrm{C}$

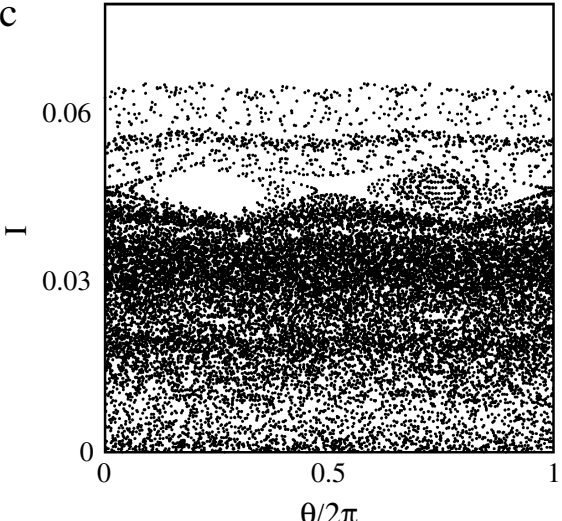

b

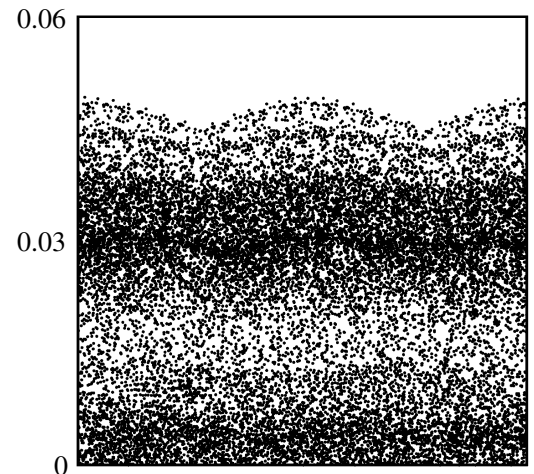

d

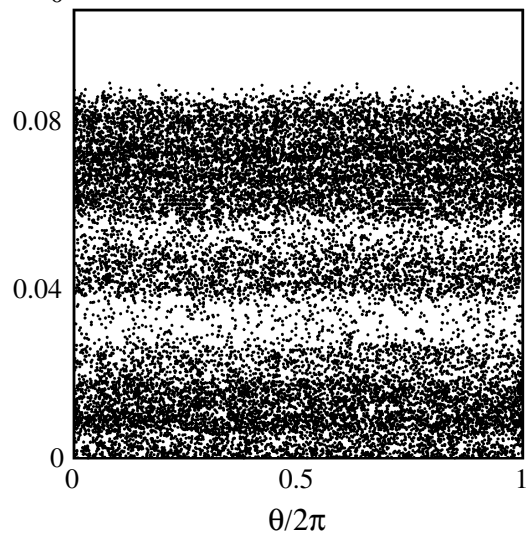

Fig. 1. Poincaré surfaces of section for map $M_{\gamma}$, see Eq. (5), with $K=10^{-3}$ and (a) $\gamma=0.2$, (b) $\gamma=0.4$, (c) $\gamma=0.6$, and (d) $\gamma=0.8$. A single initial condition with $\theta_{0}=2$ and $I_{0}=K / 100$ was iterated $2 \times 10^{4}$ times.

\section{Characterization of the discontinuous map $M_{\gamma}$}

As examples, in Fig. 1 we present Poincaré surfaces of section for map $M_{\gamma}$ with $K=10^{-3}$ and some values of $0<\gamma<1$. A single initial condition was used to construct each Poincare map. From this figure we note that, even though the phase space is not ergodic due to the sticking of trajectories along cantori (clearly observed in panels (a) and (c)), the movement is not bounded; so we expect diffusion for any combination of $K$ and $\gamma$ and, as a consequence, a monotonous growth of the action $I$.

In recent years, the dynamics of nonlinear maps, dissipative and non-dissipative, has been satisfactorily investigated by means of scaling studies of quantities like $\langle I\rangle,\left\langle I^{2}\right\rangle$, and $\omega$; see for example Refs. [7-12,20,21,23]. In particular, since $I$ is proportional to momentum and $I^{2}$ to energy, the corresponding average values of these two quantities have physical relevance because depending on the system described by our discontinuous map they may become experimentally accessible to measurements. Therefore, below we will look for the scaling properties of $\left\langle I^{2}\right\rangle$ and $\omega$ (which is a measure of the fluctuations of $I$ around its average value) as a function of $n$ as well as on the control parameters $K$ and $\gamma$.

\subsection{Scaling properties of the averaged square action $\left\langle I^{2}\right\rangle$}

We compute $\left\langle I^{2}\right\rangle$ for map (5) following two steps: First we calculate the average squared action over the orbit associated with the initial condition $j$ as

$$
\left\langle I_{n, j}^{2}\right\rangle=\frac{1}{n+1} \sum_{i=0}^{n} I_{i, j}^{2}
$$

where $i$ refers to the $i$ th iteration of the map. Note that since $I^{2}$ is proportional to energy, Eq. (6) provides indeed the average energy gain of a particle along the trajectory with initial condition $j$. Then, the average value of $I^{2}$ is defined as the average

\footnotetext{
4 Indeed, we have also performed the scaling study of $\langle I\rangle$ but we decided not to show it here since for practical purposes $\langle I\rangle$ behaves proportionally to $\sqrt{\left\langle I^{2}\right\rangle}$, therefore all conclusions obtained for $\left\langle I^{2}\right\rangle$ can be straightforwardly extended to $\langle I\rangle$.
} 


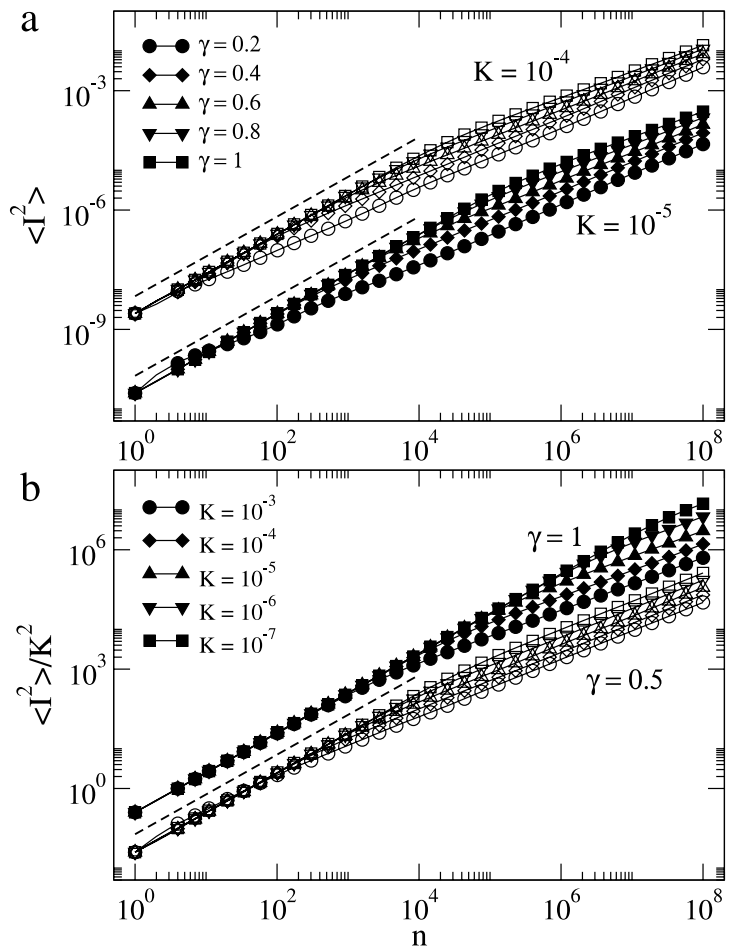

Fig. 2. (a) $\left\langle I^{2}\right\rangle$ as a function of $n$ for map $M_{\gamma}$, see Eq. (5). Full symbols (open symbols) correspond to $K=10^{-5}\left(K=10^{-4}\right)$. (b) $\left\langle I^{2}\right\rangle / K^{2}$ as a function of $n$. Full symbols (open symbols) correspond to $\gamma=1(\gamma=0.5)$. In (b) the data for $\gamma=0.5$ was displaced down by a factor of 10 for clarity. $I_{0}=K / 10^{3}$ was used. The dashed lines proportional to $n$ are plotted to guide the eye.

over $M$ independent realizations of the map (by randomly choosing values of $\theta_{0}$ ):

$$
\left\langle I^{2}\right\rangle\left(n, K, I_{0}, \gamma\right)=\frac{1}{M} \sum_{j=1}^{M}\left\langle I_{n, j}^{2}\right\rangle .
$$

In our simulations, for each combination of parameters $\left(K, I_{0}, \gamma\right)$, we consider an ensemble of $10^{3}$ different initial random phases uniformly distributed in the interval $0<\theta_{0}<2 \pi$. We have verified that larger ensembles lead to qualitatively the same results.

In Fig. 2(a) we present $\left\langle I^{2}\right\rangle$ as a function of $n$ for several values of $\gamma$ and two different values of $K: K=10^{-4}$ and $K=10^{-5}$. In addition, in Fig. 2(b) we show $\left\langle I^{2}\right\rangle$ vs. $n$ again but now for $\gamma$ fixed to 0.5 and 1 and some values of $K$. From Fig. 2 it is clear that $\left\langle I^{2}\right\rangle$ always grows as a power-law function of $n$ but the curves have an inflection point at a crossover iteration number $n_{x}^{(1)}$. So, $n_{x}^{(1)}$ separates two power-law behaviors. Moreover, by performing power-law fittings on the curves $\left\langle I^{2}\right\rangle$ vs. $n$ we determined that:

$$
\left\langle I^{2}\right\rangle \propto \begin{cases}n, & \text { for } n<n_{x}^{(1)}, \\ n^{\alpha^{(1)}}, \alpha^{(1)}<1, & \text { for } n>n_{x}^{(1)}\end{cases}
$$

We also noted from Fig. 2 that $\alpha^{(1)}$ depends on $\gamma$.

Then, in Fig. 3(a) we plot $\alpha^{(1)}$ as a function of $\gamma$. We extract $\alpha^{(1)}$ from power-law fittings of the curves $\left\langle I^{2}\right\rangle$ vs. $n$ in the asymptotic interval $10^{6}<n<10^{8}$. In this figure we present two sets of points that correspond to curves having $K=10^{-4}$ and $K=10^{-5}$. Even though the values of $\alpha^{(1)}$ extracted from curves with different $K$ change slightly for the same $\gamma$ we found the same global behavior of $\alpha^{(1)}$ vs. $\gamma$ for both values of $K$. So, we conclude that $\alpha^{(1)}$ is simply proportional to $-\gamma$, see the linear fittings performed in Fig. 3(a). To disregard the possible dependence of $\alpha^{(1)}$ on $K$, in Fig. 3(b) we present $\alpha^{(1)}$ as a function of $K$ for fixed $\gamma$. Notice that for $\gamma=0.7$ and 1 we do not report the values of $\alpha^{(1)}$ for small $K$. This is because, in these two cases, we have not clearly approached the regime $n \gg n_{x}^{(1)}$, so that we cannot accurately extract $\alpha^{(1)}$. Anyway, $\alpha^{(1)}$ vs. $K$ oscillates around a mean value $\left\langle\alpha^{(1)}\right\rangle_{K}$ that we also report in Fig. 3(a) as full circles. The linear fitting giving $\left\langle\alpha^{(1)}\right\rangle_{K} \approx 0.788-1314 \gamma$ confirms that $\alpha^{(1)} \alpha-\gamma$.

From Fig. 2 it can also be observed that the crossover iteration number $n_{x}^{(1)}$, separating the two power-law regimes of $\left\langle I^{2}\right\rangle$, depends on both $\gamma$ and $K$. To systematically extract $n_{x}^{(1)}$ from the curves $\left\langle I^{2}\right\rangle$ vs. $n$ we follow the next steps: (i) Divide 

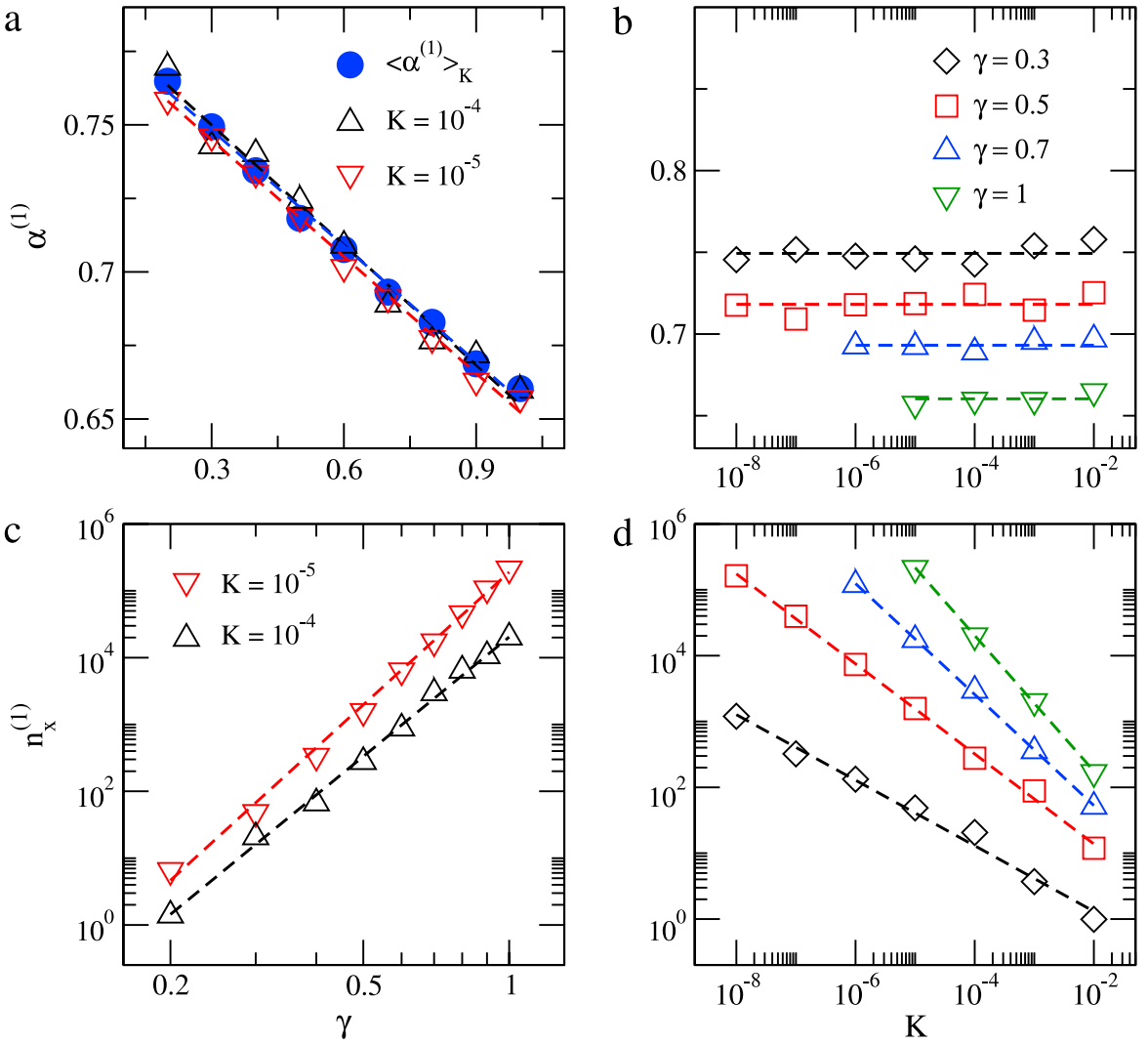

Fig. 3. (a) $\alpha^{(1)}$ as a function of $\gamma$ for $K=10^{-4}$ and $K=10^{-5}$; dashed lines are the best linear fittings $0.791-0.136 \gamma$ and $0.785-0.132 \gamma$, respectively. Also $\left\langle\alpha^{(1)}\right\rangle_{K}$ as a function of $\gamma$; the blue dashed line is the best corresponding linear fitting $0.788-1314 \gamma$. (b) $\alpha^{(1)}$ as a function of $K$ for $\gamma=0.3,0.5,0.7$, and 1 ; dashed lines are the average values of $\alpha^{(1)}$ equal to $0.7494,0.7182,0.6931$, and 0.6603 , respectively. In (a-b) $\alpha^{(1)}$ was extracted by a power-law fitting of the curves $\left\langle I^{2}\right\rangle$ vs. $n$ in the asymptotic interval $10^{6}<n<10^{8}$. (c) $n_{x}^{(1)}$ as a function of $\gamma$ for $K=10^{-4}$ and $K=10^{-5}$; dashed lines are the best power-law fittings $20662 \gamma^{5.966}$ and $189200 \gamma^{6.637}$, respectively. (d) $n_{x}^{(1)}$ as a function of $K$ for $\gamma=0.3,0.5,0.7$, and 1 ; dashed lines are the best power-law fittings $0.132 K^{-0.498}, 0.592 K^{-0.684}, 1.097 K^{-0.842}$, and $1.531 K^{-1.03}$, respectively. Same symbol (color) code as in (b). (For interpretation of the references to color in this figure legend, the reader is referred to the web version of this article.)

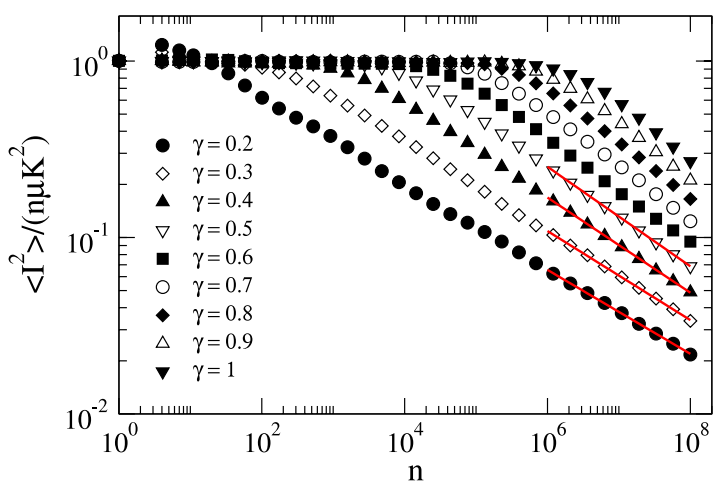

Fig. 4. $\left\langle I^{2}\right\rangle /\left(n \mu K^{2}\right)$ as a function of $n$ for map $M_{\gamma}$ with $K=10^{-6}$ and several values of $\gamma \cdot I_{0}=K / 10^{3}$ were used. We found $\mu=1 / 4$ to be the optimal value for this constant. The red lines are fittings of the form $A n^{B}$ in the interval $10^{6}<n<10^{8}$. (For interpretation of the references to color in this figure legend, the reader is referred to the web version of this article.)

$\left\langle I^{2}\right\rangle$ over $n \mu K^{2}$ such that the resulting curves are constant and equal to one for $n<n_{x}^{(1)}$, see Fig. 4; here, $\mu$ is a constant to be determined. In fact, we have observed that $\mu=1 / 4$ provides the needed effect for all combinations of $K$ and $\gamma$ we used. (ii) Perform power-law fittings of the form $A n^{B}$ in the interval $10^{6}<n<10^{8}$, where $A$ and $B$ are fitting constants; see red lines in Fig. 4. Then we define $n_{x}^{(1)}$ as the crossing point between the curves $y_{1}=A n^{B}$ and $y_{2}=1$. (iii) Evaluate $n_{x}^{(1)}$ as 

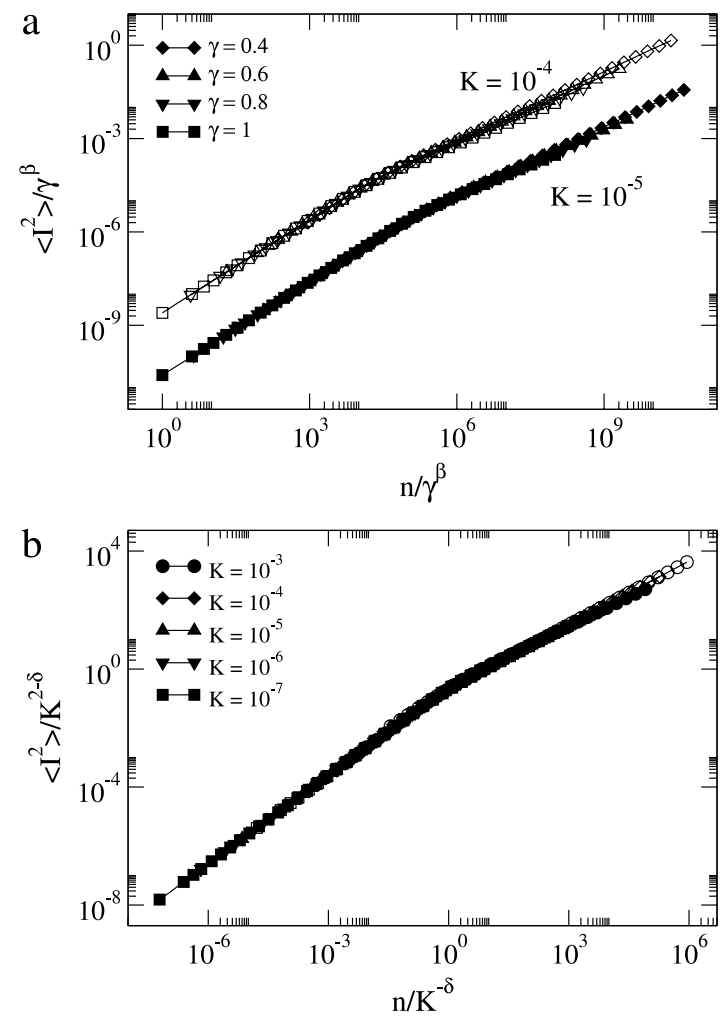

Fig. 5. (a) Scaled curves $\left\langle I^{2}\right\rangle / \gamma^{\beta}$ as a function of $n / \gamma^{\beta}$. (b) Scaled curves $\left\langle I^{2}\right\rangle / K^{2-\delta}$ as a function of $n / K^{-\delta}$. Same data as in Fig. 2 .

$n_{x}^{(1)} \equiv A^{-1 / B}$. Therefore, in Fig. 3(c) and (d) we plot $n_{x}^{(1)}$ vs. $\gamma$ and $n_{x}^{(1)}$ vs. $K$, respectively. From these figures we conclude that

$$
n_{x}^{(1)} \propto\left\{\begin{array}{l}
\gamma^{\beta} \\
K^{-\delta}
\end{array}\right.
$$

where $\beta$ and $\delta$ are scaling exponents.

Now, in Fig. 5(a) and (b) we present the scaled curves $\left\langle I^{2}\right\rangle / \gamma^{\beta}$ as a function of $n / \gamma^{\beta}$ and $\left\langle I^{2}\right\rangle / K^{2-\delta}$ as a function of $n / K^{-\delta}$, respectively. In all cases the curves fall one on top of the other up to $n_{x}^{(1)}$; for $n>n_{x}^{(1)}$ they show slightly different slopes $\alpha^{(1)}$ that depend on $\gamma$ : recall that $\alpha^{(1)} \equiv \alpha^{(1)}(\gamma)$, see Fig. 3(a).

\subsection{Scaling properties of the average standard deviation of I}

To complete our analysis here we focus on the average standard deviation of I that we define as

$$
\omega\left(n, K, I_{0}, \gamma\right)=\frac{1}{M} \sum_{j=1}^{M} \sqrt{\left\langle I_{n, j}^{2}\right\rangle-\left\langle I_{n, j}\right\rangle^{2}},
$$

where, in analogy with Eq. (6), $\left\langle I_{n, j}\right\rangle=(n+1)^{-1} \sum_{i=0}^{n} I_{i, j}$. Here, $\omega$ is a measure of the fluctuations of $I$ around the average squared action. Then, since we have already shown that $\left\langle I^{2}\right\rangle$ scales (see Fig. 5), suggesting the existence of classes of universality for the discontinuous mapping we study here, it is relevant to analyze the scaling properties of $\omega$ to find out whether $I$ is a well-behaved self-averaged variable; so the observed scaling extends to its probability distribution function.

Then, in Fig. 6 we present $\omega$ as a function of $n$ for several combinations of $\gamma$ and $K$ (in fact we have used the same parameter values of Fig. 2). In correspondence with $\left\langle I^{2}\right\rangle$ we observe two different behaviors for $\omega$ : $\omega$ increases proportional to $n^{1 / 2}$ up to a crossover iteration number $n_{x}^{(2)}$, see dashed lines in Fig. 6 . While for $n>n_{x}^{(2)}, \omega$ grows as the power-law $n^{\alpha^{(2)}}$, with $\alpha^{(2)}<1 / 2$. That is:

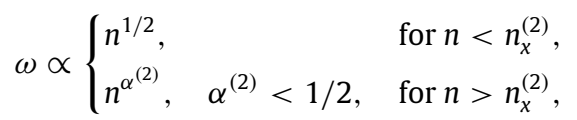

in analogy with Eq. (8). 


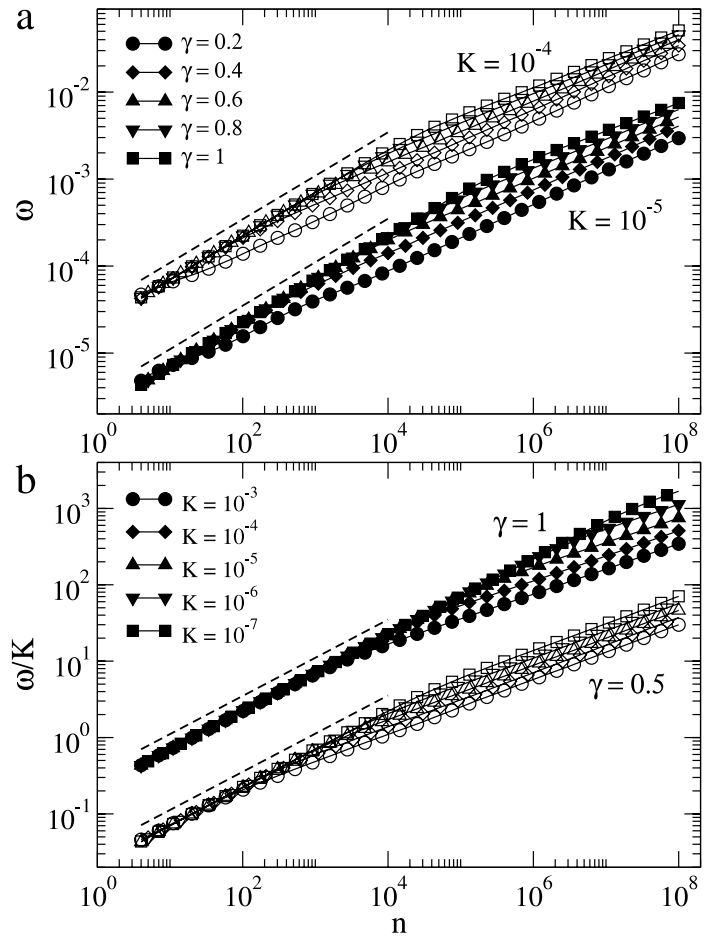

Fig. 6. (a) $\omega$ as a function of $n$ for map $M_{\gamma}$. Full symbols (open symbols) correspond to $K=10^{-5}\left(K=10^{-4}\right)$. (b) $\omega / K$ as a function of $n$. Full symbols (open symbols) correspond to $\gamma=1(\gamma=0.5)$. In (b) the data for $\gamma=0.5$ was displaced down a factor of 10 for clarity. $I_{0}=K / 10^{3}$ was used. The dashed lines proportional to $n^{1 / 2}$ are plotted to guide the eye.

We extracted the power-law exponents $\alpha^{(2)}$ and the crossover iteration numbers $n_{x}^{(2)}$ from the curves $\omega$ vs. $n$ following the procedures described in the previous Subsection to obtain $\alpha^{(1)}$ and $n_{x}^{(1)}$, respectively. Accordingly, we found that (not shown here): (i) $\alpha^{(2)} \propto-\gamma$; and (ii) $n_{x}^{(2)} \propto \gamma^{\beta}$ and $n_{x}^{(2)} \propto K^{-\delta}$ with the same $\gamma$ and $\delta$ as defined in Eq. (9). Indeed, we were able to conclude that $\alpha^{(2)}=\alpha^{(1)} / 2$ and $n_{x}^{(2)} \equiv n_{x}^{(1)}$; where the former equality is supported by the fact that $\omega \propto \sqrt{\left\langle I^{2}\right\rangle}$, see Eq. (10).

Finally, in Fig. 7 we show the scaled curves $\omega / \gamma^{\beta / 2}$ as a function of $n / \gamma^{\beta}$ and $\omega / K^{(2-\delta) / 2}$ as a function of $n / K^{-\delta}$. As expected, we observe a perfect overlap of the curves onto a single and universal plot when the curves are properly scaled.

\section{Summary}

In this work we have studied a phase transition from integrability to non-integrability for a family of two-dimensional nonlinear and discontinuous maps. The mapping is parameterized by two control parameters: (i) $K$, controlling the nonlinearity and hence a transition from integrability to non-integrability and; (ii) $\gamma$, an exponent that defines the family of discontinuous maps.

We concentrated our attention on the behavior of the average action $\langle I\rangle$ (not shown along the paper but reported in Table 1$),{ }^{5}$ the average square action $\left\langle I^{2}\right\rangle$ and the average standard deviation of $I, \omega$, as a function of the $n$th iteration of the map as well as the parameters $K$ and $\gamma$.

The critical exponents, which depend on both $K$ and $\gamma$, were obtained via extensive simulations and the scaling hypotheses were all supported by the perfect collapse of the properly scaled curves of $\left\langle I^{2}\right\rangle$ and $\omega$ for the chaotic orbits produced by the mapping; see Figs. 5 and 7. In Table 1 we summarize the scaling behaviors of $\left\langle I^{2}\right\rangle$ and $\omega$ but also of the average action $\langle I\rangle$. Notice that since the family of discontinuous maps we study here is parameterized by $\gamma$, the critical exponents that characterize the scaling functions (that depend on $\gamma$ ) define universality classes. Also, the scaling of $\omega$ allows us to conclude that the scaling of the action $I$ extends to its probability distribution function since the relative variance, which is proportional to $\omega^{2} /\left\langle I^{2}\right\rangle$, does not depend on $n$.

In particular, since $I$ is proportional to momentum and $I^{2}$ to energy, the corresponding average values of these two quantities have physical relevance because depending on the system described by our discontinuous map they may become

\footnotetext{
5 We have computed $\langle I\rangle$ as $\langle I\rangle=M^{-1} \sum_{j=1}^{M}\left\langle I_{n, j}\right\rangle$ with $\left\langle I_{n, j}\right\rangle=(n+1)^{-1} \sum_{i=0}^{n} I_{i, j}$, in analogy with Eqs. (6) and (7).
} 

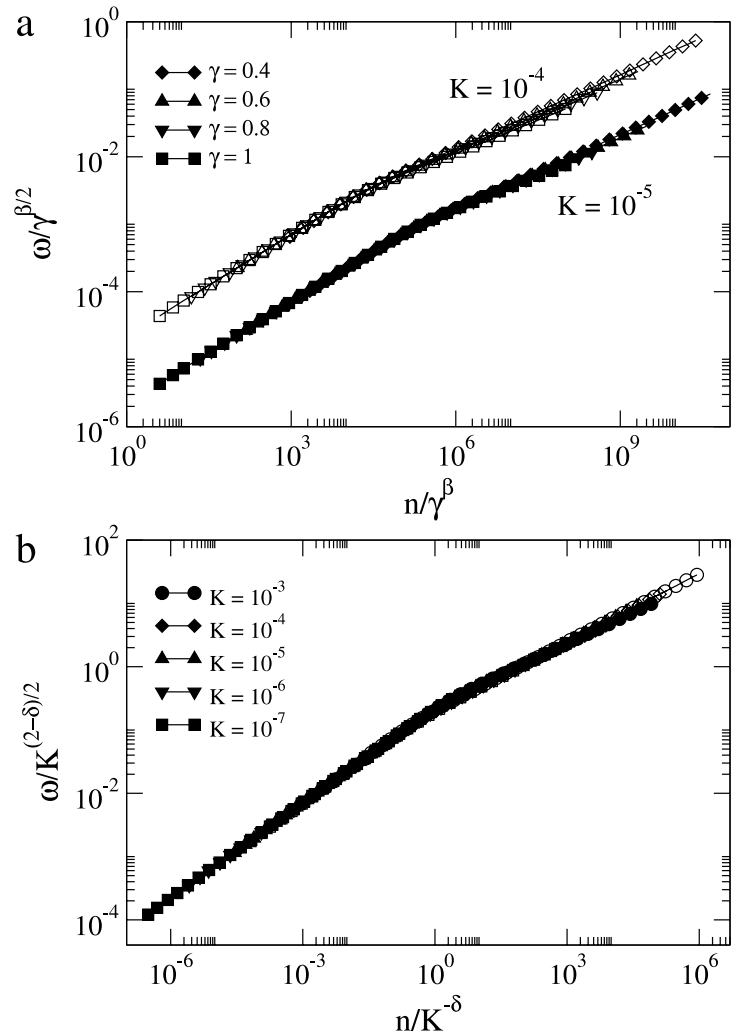

Fig. 7. (a) Scaled curves $\omega / \gamma^{\beta / 2}$ as a function of $n / \gamma^{\beta}$. (b) Scaled curves $\omega / K^{(2-\delta) / 2}$ as a function of $n / K^{-\delta}$. Same data as in Fig. 6 .

Table 1

Summary of the scaling behaviors of $\langle I\rangle$ (average momentum), $\left\langle I^{2}\right\rangle$ (average energy), and $\omega$ (average standard deviation of $I$ ) for the discontinuous map $M_{\gamma}$, see Eqs. (8) and (11); here $n_{x}=n_{x}^{(1)}=n_{x}^{(2)}$ and $\alpha=\alpha^{(1)}$ with $\alpha^{(2)}=\alpha^{(1)} / 2$. Recall that both $n_{x}$ and $\alpha$ depend on the exponent $\gamma$; see e.g. Fig. 3.

\begin{tabular}{llll}
\hline & $\langle I\rangle$ & $\left\langle I^{2}\right\rangle$ & $\omega$ \\
\hline$n<n_{x}$ & $n^{1 / 2}$ & $n$ & $n^{1 / 2}$ \\
$n>n_{x}$ & $n^{\alpha / 2}$ & $n^{\alpha}$ & $n^{\alpha / 2}$ \\
\hline
\end{tabular}

experimentally accessible to measurements. Moreover, the collapse of scaled curves of $\left\langle I^{2}\right\rangle$ onto universal curves parameterized by $\gamma$, implies that $\gamma$ is the only relevant parameter for the energy a particle may gain when diffusing under the dynamics of map $M_{\gamma}$.

Finally, we recall that for specific values of $\gamma$ our mapping acts as the discontinuous-map representation of well-known nonlinear systems (i.e., the Fermi-Ulam accelerator model and the ripple billiard model for $\gamma=1$; a time-dependent potential well for $\gamma=1 / 2$ ), thus making our study of broad applicability.

\section{Acknowledgments}

J.A.M.-B acknowledges FAPESP (Grants No. 2013/14655-9 and No. 2014/25997-0), VIEP-BUAP (Grant No. MEBJ-EXC15-I), Fondo Institucional PIFCA (Grant No. BUAP-CA-169), and CONACyT (Grants No. I0010-2014-02/246246 and No. CB-201301/220624) for support. J.A.M.-B is grateful for the warm hospitality at Departamento de Física at UNESP, Rio Claro, where this work was mostly developed. J.A.O. thanks FAPESP (Grant No. 2014/18672-8) and PROPe/FUNDUNESP/UNESP. E.D.L. is grateful to the Brazilian agencies FAPESP (Grant No. 2012/23688-5), CNPq, and CAPES.

\section{References}

[1] A.J. Lichtenberg, M.A. Lieberman, Regular and Chaotic Dynamics, Springer-Verlag, New York, 1992.

[2] B.V. Chirikov, Preprint 267, Institute of Nuclear Physics, Novosibirsk, 1969 [Engl. Trans., CERN Trans. $71-40$ (1971)].

[3] B.V. Chirikov, Phys. Rep. 52 (1979) 263.

[4] L.D. Pustylnikov, Trans. Moscow Math. Soc. 2 (1978) 1. 
[5] J.E. Howard, J. Humpherys, Physica D 80 (1995) 256.

[6] M.A. Lieberman, A.J. Lichtenberg, Phys. Rev. A 5 (1972) 1852.

[7] J.K.L. da Silva, D.G. Ladeira, E.D. Leonel, P.V.E. McClintock, S.O. Kamphorst, Braz. J. Phys. 36 (2006) 700

[8] J.A. de Oliveira, R.A. Bizao, E.D. Leonel, Phys. Rev. E 81 (2010) 046212

[9] E.D. Leonel, J.A. de Oliveira, F. Saif, J. Phys. A: Math. Theor. 44 (2011) 302001.

[10] J.A. de Oliveira, E.D. Leonel, Physica A 390 (2011) 3727.

[11] J.A. de Oliveira, C.P. Dettmann, D.R. da Costa, E.D. Leonel, Phys. Rev. E 87 (2013) 062904

[12] E.D. Leonel, P.V.E. McClintock, J. Phys. A: Math. Gen. 38 (2005) 823.

[13] L.E. Reichl, The Transition to Chaos, Springer-Verlag, New York, 2004.

[14] C. Manchein, M.W. Beims, Phys. Lett. A 377 (2013) 789.

[15] F. Borgonovi, Phys. Rev. Lett. 80 (1998) 4653.

[16] F. Borgonovi, G. Casati, B. Li, Phys. Rev. Lett. 77 (1996) 4744.

[17] G. Casati, T. Prosen, Phys. Rev. E 59 (1999) R2516.

[18] G. Casati, T. Prosen, Phys. Rev. Lett. 85 (2000) 4261.

[19] T. Prosen, M. Znidaric, Phys. Rev. Lett. 87 (2001) 114101.

[20] J.A. Mendez-Bermudez, R. Aguilar-Sanchez, Phys. Rev. E 85 (2012) 056212.

[21] R. Aguilar-Sanchez, E.D. Leonel, J.A. Mendez-Bermudez, Phys. Lett. A 377 (2013) 3216

[22] G.A. Luna-Acosta, K. Na, L.E. Reichl, A. Krokhin, Phys. Rev. E 53 (1996) 3271. See also Chapter 6 of Ref. [13].

[23] E.D. Leonel, Phys. Rev. Lett. 98 (2007) 114102;

E.D. Leonel, D.R. da Costa, C.P. Dettmann, Phys. Lett. A 376 (2012) 421

[24] G.A. Luna-Acosta, G. Orellana-Rivadeneyra, A. Mendoza-Galvan, C. Jung, Chaos Solitons Fractals 12 (2001) 349; J.L. Mateos, Phys. Lett. A 256 (1999) 113. 